\title{
BMJ Open Cost-effectiveness analysis of adding ramucirumab to the first-line erlotinib treatment for untreated EGFR-mutated metastatic non-small cell lung cancer in China
}

\author{
Qiao Liu, ${ }^{1}$ Xia Luo, ${ }^{1}$ Liubao Peng, ${ }^{1}$ Lidan Yi, ${ }^{1}$ Xiaomin Wan, ${ }^{1}$ Xiaohui Zeng, ${ }^{2}$ \\ Chongqing Tan (D) ${ }^{1}$
}

To cite: Liu Q, Luo X,

Peng $L$, et al. Costeffectiveness analysis of adding ramucirumab to the first-line erlotinib treatment for untreated EGFR-mutated metastatic non-small cell lung cancer in China. BMJ Open 2020;10:e040691. doi:10.1136/ bmjopen-2020-040691

- Prepublication history and additional material for this paper is available online. To view these files, please visit the journal online (http://dx.doi.org/10. 1136/bmjopen-2020-040691).

Received 21 May 2020

Revised 27 October 2020

Accepted 05 November 2020

Check for updates

(C) Author(s) (or their employer(s)) 2020. Re-use permitted under CC BY-NC. No commercial re-use. See rights and permissions. Published by BMJ.

${ }^{1}$ Department of Pharmacy, The Second Xiangya Hospital of Central South University, Changsha, China

${ }^{2}$ PET Imaging Center, The Second Xiangya Hospital of Central South University, Changsha, China

\section{Correspondence to} Professor Chongqing Tan; tanchongqing@csu.edu.cn and Dr Xiaohui Zeng, PET Imaging Center, the Second Xiangya Hospital of Central South University, Changsha, Hunan, China;

zengxiaohui2008@csu.edu.cn

\section{ABSTRACT}

Objective To investigate the cost-effectiveness of ramucirumab plus erlotinib compared with placebo plus erlotinib in the first-line setting for patients with EGFRmutated metastatic non-small cell lung cancer (mNSCLC) from the Chinese healthcare system perspective. Design A Markov model consisting of three health states using clinical survival data from the RELAY phase III randomised clinical trial, a lifetime horizon for costs and quality-adjusted life-years (QALYs) was constructed to analyse the cost-effectiveness of ramucirumab plus erlotinib. One-way and probabilistic sensitivity analyses were performed to evaluate the robustness of the model. Additional price reduction scenario analyses were performed.

Setting The Chinese healthcare system perspective. Participants A hypothetical Chinese cohort of patients with confirmed previously documented ex19del or Leu858Arg mutation stage IV NSCLC, and without known epidermal growth factor receptor (EGFR) Thr790Met mutation and central nervous system metastases.

Interventions Ramucirumab plus erlotinib versus placebo plus erlotinib.

Primary outcome measure Costs, QALYs, incremental cost-effectiveness ratio (ICER).

Results In base-case analysis, ramucirumab plus erlotinib yield an additional 4.21 QALYs at a cost of $\$ 540590$, resulting in an ICER of $\$ 128302 /$ QALY. In price reduction scenario analysis, the ICER ( $\$ 65$ 227/QALY) was decreased significantly when the National Reimbursement Drug List (NRDL) negotiation was available for ramucirumab, and the ICER (\$131 554/QALY) was increased slightly when the NRDL negotiation was unavailable for erlotinib. Sensitivity analyses demonstrated our results to be most sensitive to the unit cost of ramucirumab $(10 \mathrm{mg} / \mathrm{kg})$, and more than $52.1 \%$ reduction in the price of ramucirumab resulted in the ICER under the willingness-to-pay threshold set for affluent regions (\$70 353/QALY).

Conclusions Ramucirumab plus erlotinib is unlikely to be cost-effective for patients with untreated EGFR-mutated mNSCLC in China. Reducing the price of ramucirumab through the National Healthcare Security Administration negotiation was found to be the most realistic action to improve cost-effectiveness.

\section{Strengths and limitations for this study}

- The trial-based Markov model can make a longterm projection based on the latest clinical trial data, which usually have short periods.

- The Markov model was able to simulate the treatment, survival and death of advanced non-small cell lung cancer, which makes up the limitation exist in commonly used meta-analytic techniques.

- We applied two willingness-to-pay thresholds in the model, reflecting the cost-effectiveness of ramucirumab plus erlotinib versus placebo plus erlotinib in both high-income and resource-constrained regions of China.

- The trial-based economic analysis should be interpreted with caution because the worldwide trial may not fully conform to the clinical pathway and treatment pattern in China.

- A potential for inherent bias in our Markov model was that several local data on costs and utilities were not available.

\section{INTRODUCTION}

Lung cancer remains the most prevalent malignancy, ${ }^{1}$ as well as the leading cause of cancer-related deaths in China. ${ }^{2}$ Nonsmall cell lung cancer (NSCLC) manifests the majority (nearly $85 \%$ ) of primary lung cancers, ${ }^{34}$ and up to $46 \%$ of NSCLC cases diagnosed beyond early stages. ${ }^{5}$ Epidermal growth factor receptor (EGFR) mutations occur at frequencies of approximately $30 \%-50 \%$ in Asian patients with NSCLC, of which in-frame deletion within exon 19 (ex19del) accounts for $44 \%$ and the single point mutation in exon 21 (Leu858Arg) accounts for $41 \%{ }^{6}$

Historically, the most common form of personalised treatment for EGFR mutationdriven NSCLC has been the targeted therapy with small-molecule tyrosine 
kinase inhibitors (TKIs), which are recommended as the first-line standard-of-care, because of their major improvement over traditional chemotherapy in longing survival. ${ }^{7-10}$ Clinical evidence over the past few years has shown that the median progression-free survival (PFS) with TKIs for advanced disease ranges from about 1 year for first-generation TKIs (gefitinib and erlotinib), to 18.9 months for third-generation TKIs (osimertinib). ${ }^{7-9} 1112$ However, EGFR TKIs are related to inevitably treatment resistance, which eventually leads to the loss of clinical benefits. Additionally, although great progress has been made in immune checkpoint inhibitors in recent years, ${ }^{13}$ their role in EGFR-mutated disease is poor, ${ }^{14}$ and treatment options for patients who have exhausted from these targeted therapies are generally limited. ${ }^{15} 16$ Thus, there is an ongoing unmet need for new treatment strategies, such as EGFR TKI-based combination therapy, to provide the best chance of long-time PFS, and therefore prolong remission and control tumour.

Increasing preclinical and clinical trials of first-line treatment of EGFR-mutated NSCLC has revealed better clinical outcomes for the dual blockade of the EGFR and vascular endothelial growth factor (VEGF) pathways, than for inhibition of the EGFR pathway alone. ${ }^{17-20}$ Ramucirumab, a fully human monoclonal IgG1 antibody, binds specifically to vascular endothelial growth factor receptor 2 (VEGFR-2) with high affinity. ${ }^{21}$ Therefore, ramucirumab has more extensive antitumor activity in contrast to other VEGF inhibitors. A very recent, phase III RELAY trial supported the potential of adding ramucirumab to the first-line erlotinib treatment. Compared with placebo plus erlotinib, ramucirumab plus erlotinib provided superior PFS in patients with untreated EGFRmutated metastatic NSCLC (mNSCLC) (19.4 months vs 12.4 months; HR $=0.59,95 \%$ CI: $0.46-0.76, p<0.0010){ }^{22}$ Notably, a consistent PFS benefit was observed in patients with a baseline ex19del and Leu858Arg mutations. Although there has been a general uptrend in the incidence of several treatment-emergent adverse events for the combination strategy, the increase has been mainly limited to grade 1-2 events.

The significantly higher efficacy of ramucirumab plus erlotinib in comparison with placebo plus erlotinib has inspired us that the dual inhibition of EGFR and VEGF pathways appears to be a better alternative for patients with untreated EGFR-mutated mNSCLC. ${ }^{22}$ However, in mainland China, ramucirumab, the novel VEGFR-2 specific inhibitor with prohibitive price, has not been licensed. Instead, erlotinib has been approved for the first-line treatment of EGFR-mutated mNSCLC. The retail price of erlotinib in China was set by the National Healthcare Security Administration (NHSA) through National Reimbursement Drug List (NRDL) negotiation, which is $70 \%$ lower than its original price. ${ }^{23}$ Cost-effectiveness analyses of an expensive new treatment strategy is necessary before it is widely used, especially in health resourcelimited setting. In order to better determine the role of the dual blockade of the EGFR and VEGF pathways in first-line metastatic EGFR-mutated NSCLC, we performed a cost-effectiveness analysis to compare ramucirumab plus erlotinib with placebo plus erlotinib, from the perspective of Chinese healthcare. Information on significant effectiveness incurred by ramucirumab could shed light on policy decision towards its listing in China.

\section{MATERIAL AND METHODS \\ Analytical overview and model structure}

A Markov model was constructed to evaluate the costeffectiveness associated with the two first-line strategies for patients with mNSCLC: (1) ramucirumab $10 \mathrm{mg} / \mathrm{kg}$ every 2 weeks, plus oral erlotinib $150 \mathrm{mg} /$ day; (2) intravenous placebo plus oral erlotinib. Our study is a trial-based economic assessment that used clinical data mainly from the RELAY phase III clinical trial. Therefore, a hypothetical cohort consistent with the RELAY trial population (patients with confirmed previously documented ex19del or Leu858Arg mutation stage IV NSCLC, and without known EGFR Thr790Met mutation and central nervous system (CNS) metastases, and the initial age for all patients was 65 years old) was created in the model to simulate the clinical efficacy reflected in the RELAY clinical trial. This model-based economic evaluation relied on a literature review, and it is exempt from the institutional research ethics board approval.

The course of mNSCLC was simulated by a Markov model, including three mutually exclusive health states (figure 1): PFS, progression survival (PS) and death. The Markov cycle length was 2 weeks, and the time horizon was lifetime. Half-cycle correction was adopted in the model. All hypothetical patients were in the PFS state initially, and randomly treated with first-line ramucirumab-erlotinib or placebo-erlotinib until Response Evaluation Criteria In Solid Tumors (RECIST) defined progression, or unacceptable toxicity. As reported in the RELAY trial, at the date cut-off, 29\% (64 of 224) of patients in ramucirumab-erlotinib arm and 19\% (43 of 225) in placebo-erlotinib arm were still under treatment. After RECIST-defined progression, we modelled patients as receiving type of subsequent line of therapy consistent with those detail in the RELAY trial. Subsequent line of therapy that included chemotherapy, EGFR-TKI targeted therapy and immunotherapy was allowed as long as there was continued benefit as judged by the investigator, when first subsequent line of therapy was failed, second subsequent line of therapy was administered at the discretion of the investigator. According to the RELAY trial, over half of patients received first subsequent line of therapy $(54 \%$ in ramucirumab-erlotinib and $69 \%$ in placebo-erlotinib, respectively), and more patients were administered second subsequent line of therapy in ramucirumaberlotinib arm than placebo-erlotinib arm (28\% vs $34 \%$, respectively). ${ }^{22}$ Patients who had not received subsequent therapy were treated with the best supportive care (BSC) based on current clinical guidelines in china. ${ }^{24}$ 


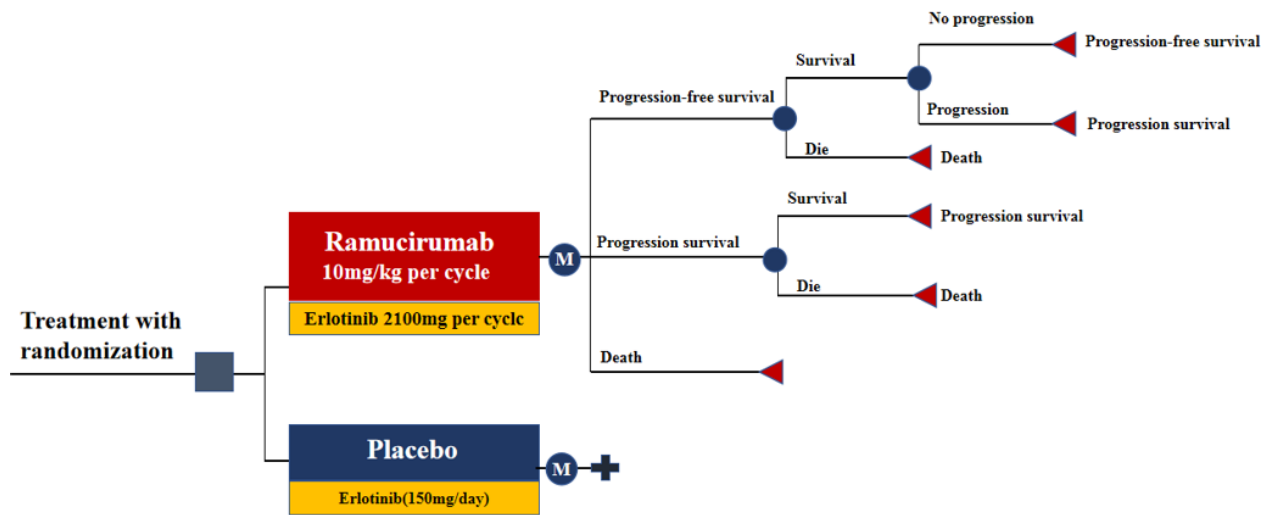

The primary outcomes in the model were as follows: the total cost, life years (LYs), quality-adjusted lifeyears (QALYs) and incremental cost-effectiveness ratios (ICERs). Cost and effectiveness were discounted at 3\% annually. Due to the imbalance of economic development among different regions in china, we selected $\$ 30$ 363/ QALY as the willingness-to-pay (WTP) threshold value for general regions and $\$ 70353 /$ QALY for affluent regions. ${ }^{25}$

\section{Model survival and progression risk estimates}

The clinical effectiveness data associated with ramucirumab-erlotinib and placebo-erlotinib were available from the phase III RELAY trial, at the updated data cutoff (1 February 2018). ${ }^{22}$ GetData Graph Digitizer software package (V.2.26; http://www.getdata-graph-digitizer. com/download.php) was used to extract the PFS and overall survival (OS) data points from the investigatorassessed Kaplan-Meier (KM) curves, then four commonly used parametric survival models were fitted, including Weibull, exponential, log-logistic and log-normal distributions. The exponential survival distribution was chosen to independently fit the digitised KM plots of the two firstline treatments, as it provided the optimal fit based on statistical goodness-of-fit test (Bayesian information criterion (BIC) and Akaike's information criterion (AIC)), visual fit and clinical rationality (see online supplemental appendix 1). The exponential distribution parameters, hazard rate $\left(\lambda_{\mathrm{OS}}\right.$ and $\left.\lambda_{\mathrm{PFS}}\right)$, was estimated by $\mathrm{R}$ software (V.3.3.1, http://www.r-project.org). Table 1 shows the final estimated exponential parameters. For the validation purpose, the predicted OS and PFS curves were compared with the investigated KM curves (see online supplemental appendix 1).

The time-dependency transition probabilities of death were calculated from the following formula:

$$
t p\left(t_{u}\right)=\exp \left\{\lambda_{o s}(t-u)-\lambda_{o s} t\right\}(\lambda>0),
$$

The transition probabilities of PFS at time $t$ were calculated from the following formula:

$$
t p\left(t p\left(t_{u}\right)=\frac{\exp \left\{\lambda_{P F S}(t-u)-\lambda_{P F S} t\right\}}{\exp \left\{\lambda_{O S}(t-u)-\lambda_{O S} t\right\}}(\lambda>0),\right.
$$

where $t_{u}$ represents the arrival state after $u$ Markov cycles, and $t$ is calculated as integer multiple of Markov cycle length. ${ }^{26}$

\section{Utility estimates}

QALYs in the model were estimated by weighting the patient's life years with health utility value. Health utility values of PFS and PS states were derived from a previously published international study that capture utilities for mNSCLC in six countries, including China. ${ }^{27}$ According to the study, utility decrements were found for treatmentrelated grade III/IV toxicities. Therefore, the current analysis calculated the utility value in PFS states based on the risk of adverse events reported in the RELAY trial, and the corresponding utility values were as follows: PFS (0.815), PFS plus diarrhoea (0.746), PFS plus hypertension (0.773), PFS plus rash (0.720), PFS plus nausea/ vomiting (0.695), PFS plus fatigue (0.750) and PFS plus neutropenia $(0.621) .{ }^{27}$ Utility values used in the model are listed in table 1 .

\section{Cost estimates}

The cost data were estimated from the perspective of Chinese healthcare, and only direct medical costs were considered in this model, including drug, management of serious adverse effects (SAEs) (grade III/IV adverse effects), routine follow-up in PFS state, subsequent therapy in PS sate, BSC and terminal care cost (table 1). The unit cost for erlotinib (2100 mg per cycle) was based on the latest reimbursement price, negotiated by the NHSA with pharmaceutical companies in July 2017. ${ }^{23}$ The unit cost of ramucirumab $(10 \mathrm{mg} / \mathrm{kg}$ per cycle) was 
Table 1 Base cases, ranges and distributional assumptions of parameters

\begin{tabular}{|c|c|c|c|c|}
\hline Parameter & Base-case & Range & Distribution & Source \\
\hline \multicolumn{5}{|l|}{ Costs (\$) } \\
\hline Ramucirumab (10 mg/kg per unit) & 100.5 & $43.5-100.5$ & Fixed & Local charge \\
\hline Erlotinib (2100 mg per unit)* & 115.6 & $115.6-385.3$ & Fixed & Local charge \\
\hline Routine follow-up per unit $†$ & 37.1 & $27.8-46.3$ & Lognormal & Wu et $a l^{31}$ \\
\hline Subsequent therapy in PS state per unitł & 558.4 & $462.0-648.9$ & Lognormal & Zeng et $a^{32}$ \\
\hline BSC per unit§ & 225.0 & $105.8-529.1$ & Lognormal & Wu et $a l^{31}$ \\
\hline Terminal phase cost per unitๆ & 1751.9 & $1527.9-1977.7$ & Lognormal & Zeng et $\left.a\right|^{32}$ \\
\hline Hypertension per event & 12.9 & $11.6-14.2$ & Lognormal & Wu et $\left.a\right|^{29}$ \\
\hline Diarrhoea per event & 5.18 & $4.14-6.22$ & Lognormal & Lu et $a l^{28}$ \\
\hline \multicolumn{5}{|l|}{ Risk for SAEs } \\
\hline Diarrhoea in ramucirumab arm & 0.072 & $0.058-0.086$ & Beta & Nakagawa et al ${ }^{22}$ \\
\hline Diarrhoea in placebo arm & 0.013 & $0.010-0.016$ & Beta & Nakagawa et $a^{22}$ \\
\hline Hypertension in ramucirumab arm & 0.235 & $0.188-0.282$ & Beta & Nakagawa et al ${ }^{22}$ \\
\hline Hypertension in placebo arm & 0.053 & $0.042-0.064$ & Beta & Nakagawa et al ${ }^{22}$ \\
\hline Rash in ramucirumab arm & 0.009 & $0.007-0.011$ & Beta & Nakagawa et al ${ }^{22}$ \\
\hline Rash in placebo arm & 0.022 & $0.018-0.026$ & Beta & Nakagawa et al ${ }^{22}$ \\
\hline Vomiting in ramucirumab arm & 0.009 & $0.007-0.011$ & Beta & Nakagawa et $a l^{22}$ \\
\hline Vomiting in placebo arm & 0.004 & $0.003-0.005$ & Beta & Nakagawa et al22 \\
\hline Fatigue in ramucirumab arm & 0.014 & $0.011-0.017$ & Beta & Nakagawa et al ${ }^{22}$ \\
\hline Fatigue in placebo arm & - & - & Beta & Nakagawa et al ${ }^{22}$ \\
\hline Neutropenia in ramucirumab arm & 0.027 & $0.022-0.032$ & Beta & Nakagawa et al22 \\
\hline Neutropenia in placebo arm & 0.009 & $0.007-0.011$ & Beta & Nakagawa et al ${ }^{22}$ \\
\hline \multicolumn{5}{|l|}{ Health utility values } \\
\hline PFS state & 0.815 & $0.652-0.978$ & Beta & Nafees et $\left.a\right|^{27}$ \\
\hline PS state & 0.321 & $0.257-0.385$ & Beta & Nafees et $a^{27}$ \\
\hline PFS plus diarrhoea & 0.746 & $0.597-0.895$ & Beta & Nafees et $a^{27}$ \\
\hline PFS plus hypertension & 0.773 & $0.618-0.928$ & Beta & Nafees et $a^{27}$ \\
\hline PFS plus rash & 0.720 & $0.576-0.846$ & Beta & Nafees et $a^{27}$ \\
\hline PFS plus nausea/vomiting & 0.695 & $0.556-0.834$ & Beta & Nafees et $a l^{27}$ \\
\hline PFS plus fatigue & 0.750 & $0.600-0.900$ & Beta & Nafees et $a^{27}$ \\
\hline PFS plus neutropenia & 0.621 & $0.497-0.745$ & Beta & Nafees et $a^{27}$ \\
\hline \multicolumn{5}{|l|}{ Distribution parameters } \\
\hline Ramucirumab, OS, scale (exponential) & 0.003728 & - & Fixed & Estimated \\
\hline Placebo, OS, scale (exponential) & 0.004 & - & Fixed & Estimated \\
\hline Ramucirumab, PFS, scale (exponential) & 0.02617 & - & Fixed & Estimated \\
\hline Placebo, PFS, scale (exponential) & 0.01844 & - & Fixed & Estimated \\
\hline Discount rate (\%) & 3 & $0-8$ & Fixed & Guan et $\left.a\right|^{33}$ \\
\hline Patient weight $(\mathrm{kg})$ & 65 & $52-78$ & Fixed & Lu et $a l^{28}$ \\
\hline
\end{tabular}

*The price of erlotinib was set by the National Healthcare Security Administration (NHSA), for patients with EGFR (epidermal growth factor receptor) -mutated metastatic non-small cell lung cancer (mNSCLC) treated with erlotinib, $70 \%$ of the cost would be paid by China's basic medical insurance.

†The cost of routine follow-up included the cost of outpatient physician visit, laboratory tests and examinations.

$\ddagger$ According to RELAY trial, subsequent therapy referred to the treatment beyond the point of RECIST-defined progression, and included chemotherapy, EGFR-TKI targeted therapy and immunotherapy.

$\S B S C$ referred to the intervention of clinical symptoms caused by cancer, including anti-inflammatory treatment, analgesic treatment, antiemetic treatment, thoracic and abdominal puncture decompression, blood transfusion and nutritional support and so on.

IThe terminal phase cost referred to the cost of palliative end-of-life.

BSC, best supportive care; OS, overall survival; PFS, progression-free survival; PS, progression survival; RECIST, Response Evaluation Criteria In Solid Tumors; SAEs, serious adverse effects; TKI, tyrosine kinase inhibitor. 
retrieved using the latest retail price driven from ChinaHong Kong, as a result of the absence of ramucirumab in the Chinese mainland market. In calculating dosage amounts, a base-case patient with body weight of $65 \mathrm{~kg}$ (range: $52-78 \mathrm{~kg}$ ) was assumed in the model. ${ }^{28}$ In order to improve estimates accuracy of our model, the total costs of ramucirumab and erlotinib for each strategy were adjusted according to the median relative dose intensity reported in RELAY trial (see online supplemental appendix 2).

The costs of SAEs with $\geq 5 \%$ difference in incidence between the two arms were considered in the model, including hypertension, diarrhoea, dermatitis acneiform. Based on the Chinese oncologists' common opinion, dermatitis acneiform does not require additional treatment. Therefore, the costs of dermatitis acneiform were excluded. The costs related to SAEs were calculated by multiplying the incidence of SAEs by the costs of managing SAEs per event. The incidence of SAEs was derived from previous study. ${ }^{29} 30$

Other costs were obtained from published literature. ${ }^{31}{ }^{32}$ All costs are reported in 2019 US dollars. Considering that costs related to Chinese healthcare are stable under central control by the government, the current analysis did not consider the inflation of the costs from different base years.

\section{Sensitivity analysis}

Considering the uncertainty bound to model parameters, a series of sensitivity analyses were performed to evaluate the robustness of the base-case results. In the one-way sensitivity analyses (OSA), all parameters varied over a priori defined range independently (shown in table 1), while the others were fixed to explore the sensitivity of the finding to plausible variations in specific parameters. The ranges of parameters were mainly come from published literature, ${ }^{29-33}$ or were assumed to vary within $\pm 20 \%$ of the base-case value. In view of a series of negotiations on oncology drugs launched by China's NHSA, the average price reduction for new anticancer drugs was as high as $56.7 \%$, $^{23}$ the cost ranges of ramucirumab (10 $\mathrm{mg} / \mathrm{kg}$ per cycle) and erlotinib (2100 mg per cycle) were not assumed to vary within $\pm 20 \%$ of the base-case value in the current analysis. For the cost range of ramucirumab $(10 \mathrm{mg} / \mathrm{kg}$ per cycle), given a likely scenario that NHSA negotiations were available for ramucirumab, our analyses were conducted based on the price variation from $\$ 43.5$ (56.7\% of reduction, the average price reduction through NRDL negotiation) to $\$ 100.5$ (the latest retail price). For the cost range of erlotinib (2100 mg per cycle), given a likely scenario that NRDL negotiation was unavailable for erlotinib, our analyses were conducted based on the price variation from $\$ 115.6$ (70\% reduction, the latest reimbursement price set by NHSA) to $\$ 385.3$ (the latest retail price). The results of OSA were visualised by a tornado diagram.

The Monte Carlo simulation probabilistic sensitivity analyses (PSA) were performed by running 10000 iterations, to test the influence of uncertainty in the model parameters on the ICERs for each strategy. Each iteration, we varied all parameters simultaneously, except for specific parameters such as ramucirumab cost $(10 \mathrm{mg} / \mathrm{kg}$ per unit), erlotinib cost (2100 mg per unit), distribution parameters, discount rate and patient weight. The parameters were sampled from the set statistical distributions, including log-normal distributions for cost parameters, beta distributions for risks and utilities. Cost-effectiveness acceptability curves were created to illustrate the probabilities of each treatment strategy being cost-effective with respect to a given scenario, such as a wide range of WTP thresholds and different oncology drug prices. The basecase values, ranges and distributions of model parameters are listed in table 1.

\section{Patient and public involvement}

No patients or public were involved in the study.

\section{RESULTS}

\section{Base-case results}

The model showed that ramucirumab plus erlotinib in the first-line treatment for patients with untreated ex19del or Leu858Arg mutated mNSCLC result in substantial health benefits at incremental costs. The estimated mean PFS time and life expectancy for patients receiving ramucirumab plus erlotinib were 2.93 LYs and 9.04 LYs, respectively, which were $2.16 \mathrm{LYs}$ and $7.85 \mathrm{LYs}$ more than patients receiving placebo plus erlotinib. Adjusted for utilities, the ramucirumab plus erlotinib treatment added costs of $\$ 540590$ ( $\$ 554776$ vs $\$ 14185$ ) and yielded an addition QALYs of 4.21 (5.22 QALYs vs 1.01 QALYs), compared with the placebo plus erlotinib treatment. The ICER per LY gained and per QALY gained of the ramucirumab-erlotinib arm versus the placebo-erlotinib arm were $\$ 54015$ and $\$ 128302$, respectively (table 2 ).

The base-case results based on whether NRDL negotiation was available for erlotinib and ramucirumab were also reported in the current analysis (see online supplemental appendix 3).

\section{One-way sensitivity analysis}

The top five parameters with the greatest influence on the ICER for ramucirumab plus erlotinib strategy were (1) the unit cost of ramucirumab $(10 \mathrm{mg} / \mathrm{kg}) ;(2)$ discount rate; (3) patient weight; (4) the utility of PS and (5) the cost of BSC (figure 2). More than $52.1 \%$ reduction in the price of ramucirumab resulted in the ICER under the WTP threshold set for affluent regions ( $\$ 70$ 353/QALY). Other parameters, such as utility values, and other direct medical costs had a moderate effect, and the risk of SAEs had a minor effect on the ICER. The result of OSA showed that all parameters varying over a plausible range, except the unit cost of ramucirumab $(10 \mathrm{mg} / \mathrm{kg})$, failed to make ICER lower than the WTP threshold selected for affluent regions, and none of these parameters showed a 
Table 2 Summary of cost and outcome results in the base-case analysis

\begin{tabular}{|c|c|c|c|}
\hline Item & Ramucirumab-erlotinib & Placebo-erlotinib & Difference \\
\hline \multicolumn{4}{|l|}{ Mean LYs } \\
\hline PFS state & 2.93 & 0.77 & 2.16 \\
\hline Total & 11.97 & 1.96 & 10.01 \\
\hline \multicolumn{4}{|l|}{ Mean QALYs } \\
\hline PS state & 2.90 & 0.38 & 2.52 \\
\hline Total & 5.22 & 1.01 & 4.21 \\
\hline \multicolumn{4}{|l|}{ Cost $(\$)$} \\
\hline Drug costs in PFS state* & 476536 & 2187 & 479349 \\
\hline Routine follow-up cost in PFS state & 2801 & 728 & 2073 \\
\hline BSC costs in PS state & 38050 & 4589 & 33461 \\
\hline Terminal phase costs in dead state & 474 & 826 & -352 \\
\hline Total & 554776 & 14186 & 540590 \\
\hline \multicolumn{4}{|l|}{ ICER (\$) } \\
\hline Per LY & & & 54015 \\
\hline Per QALY & & & 128302 \\
\hline
\end{tabular}

*The costs of first-line ramucirumab plus erlotinib and placebo plus erlotinib were calculated based on clinical usage and dosage in RELAY trial, and then were adjusted according to median duration of therapy and median relative dose intensity.

†Subsequent therapy costs in PS state were estimated based on the proportion of patients in the first subsequent line of therapy and second subsequent line of therapy reported in RELAY trial.

BSC, best supportive care; ICER, incremental cost-effectiveness ratio; LY, life-year; PFS, progression-free survival; PS, progression survival;

QALY, quality-adjusted life-year; SAEs, serious adverse effects.

potential to reduce the ICER below the WTP threshold selected for general regions.

\section{Probabilistic sensitivity analysis}

The results of PSA are presented in figures 3 and 4. When the WTP threshold was 70 353/QALY, the proportions of simulations with cost-effectiveness for ramucirumab plus erlotinib strategy were $23.5 \%$, while when the WTP threshold was $\$ 30363 / \mathrm{QALY}$, the proportions dropped to $2.6 \%$ (figure 3 ). The acceptability curve also suggested that the proportions of simulations with cost-effectiveness for ramucirumab plus erlotinib were increased with the increasing WTPs, at a very high threshold (>\$40 000/QALY), more than $90 \%$ of simulations achieve cost-effectiveness. Figure 4 demonstrates that the likelihood of ramucirumab plus erlotinib being cost-effective increased with the decrease of the unit cost of ramucirumab, when the unit cost of ramucirumab decreased by $56.7 \%$, the probabilities of ramucirumab plus erlotinib being cost-effective were $6.1 \%$ and $53.4 \%$ at the WTP threshold of $\$ 30353 /$ QALY and the WTP threshold of $\$ 70353 /$ QALY, respectively. Additionally, we provided more detailed results of PSA in online supplemental appendix 4 .

\section{DISCUSSION}

To the best of our knowledge, the study is the first economic analysis of ramucirumab plus erlotinib versus placebo plus erlotinib for patients with previously untreated EFGR-mutated mNSCLC without known EGFR Thr790Met mutation and CNS metastases, and our results have reference significance for further promoting the NRDL negotiation in China. The main finding of the current analysis was that ramucirumab plus erlotinib could improve health outcomes (5.22 QALYs vs 1.01 QALYs) with a substantial augmentation of cost $(\$ 554$ 776 vs \$14 185) compared with placebo plus erlotinib, resulting in an average ICER of \$128 302/QALY, which is higher than the two WTP thresholds selected for the current study. From the perspective of Chinese healthcare, the ramucirumab plus erlotinib strategy was unlikely to be cost-effective.

The most influential parameter in our model was the unit cost of ramucirumab, which was mainly responsible for the unfavourable ICER of ramucirumab plus erlotinib versus placebo plus erlotinib. Fortunately for Chinese patients with cancer, a growing number of oncology drugs have been negotiated by NHSA to reduce the price since $2016 .{ }^{23}$ When the NRDL negotiation 


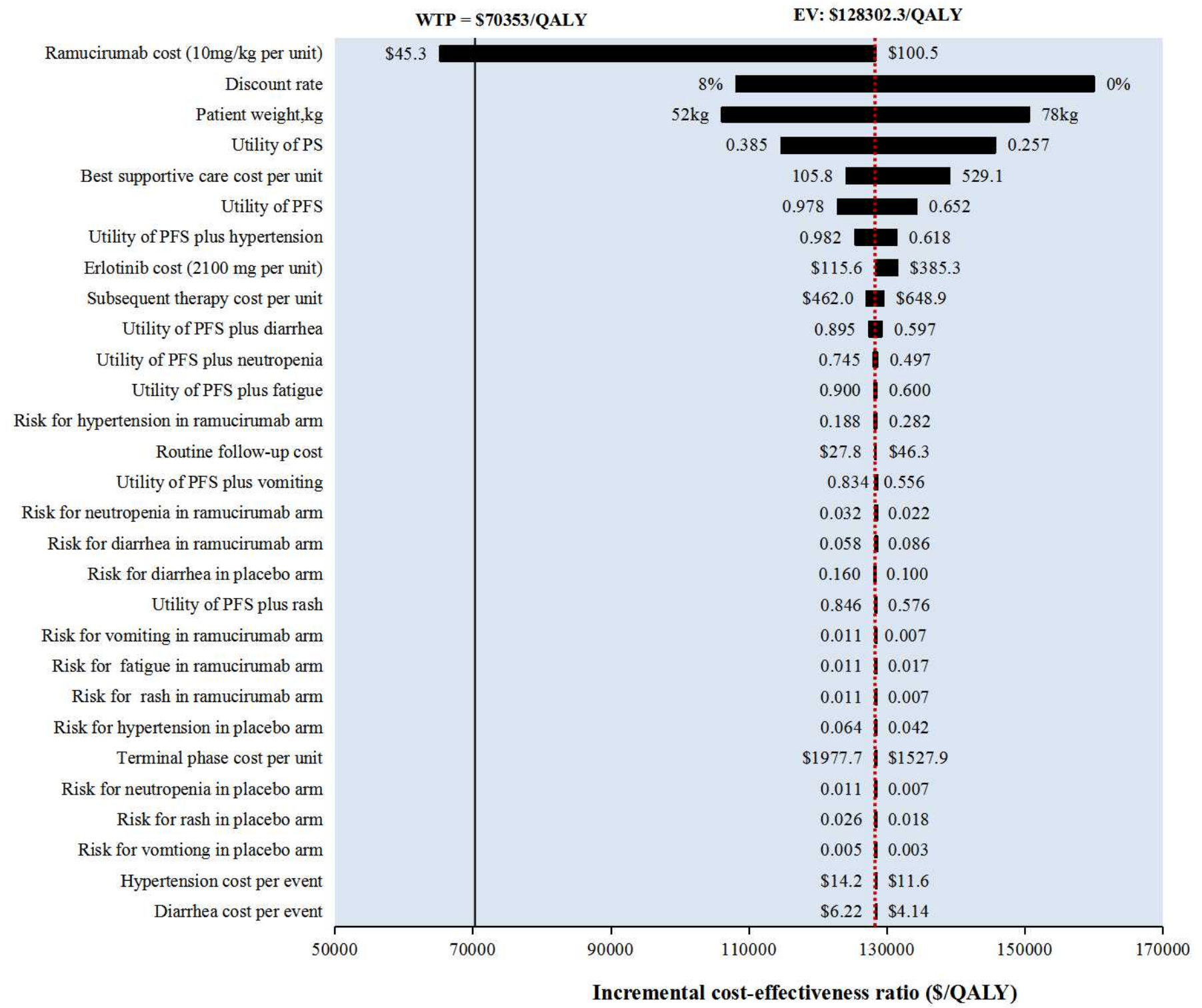

Figure 2 Tornado diagram of the one-way sensitivity analysis revealing variables' influence on the ICER. The red dotted line represents the ICER of \$128 302/QALY from the base-case results. The solid black line represents the WTP of \$70 353/QALY for affluent regions in China. ICER, incremental cost-effectiveness ratio; PFS, progression-free survival; PS, progression survival; QALY, quality-adjusted life-year; WTP, willingness-to-pay threshold.

was available for ramucirumab, the ramucirumab plus erlotinib strategy might be a cost-effective therapeutic strategy alternative to erlotinib monotherapy, given that the ICER (\$65 227/QALY) was substantially lower than the WTP threshold selected for the affluent regions $(\$ 70$ 353/QALY) in China. Thus, negotiating ramucirumab might be a feasible way to achieve favourable economic outcomes. From a more far-sighted perspective, NRDL negotiation will be the most attainable approach for integrating medical and health resource in China, through which patients with cancer can be provided with better treatment at lower cost.

In the current analysis, we also evaluated the impact of the price of erlotinib, another oncology drug, on economic outcomes. The OSA also pointed out that the ICERs were almost unaffected when the unit cost of erlotinib varied across a plausible range. When the NRDL negotiation was unavailable for erlotinib, the base-case analysis showed that ramucirumab plus erlotinib strategy yielded an unfavourable ICER of $\$ 131554$ /QALY compared to placebo plus erlotinib strategy, which was slightly higher than our primary economic outcomes. One plausible explanation for this finding is that the dose and schedule of erlotinib in the two competing strategies were exactly the same.

The PSA found a higher likelihood that ramucirumab plus erlotinib would be cost-effective at a higher WTP threshold, which were generally coherent with our previous study. ${ }^{34}$ Given the fact that the Chinese economy is highly unbalanced among 34 province-level administrative units, the per capita gross domestic product (GDP) extended from \$4727 in Gansu Province to \$29 510 in Shenzhen city in 2019. Therefore, in the current analysis, 


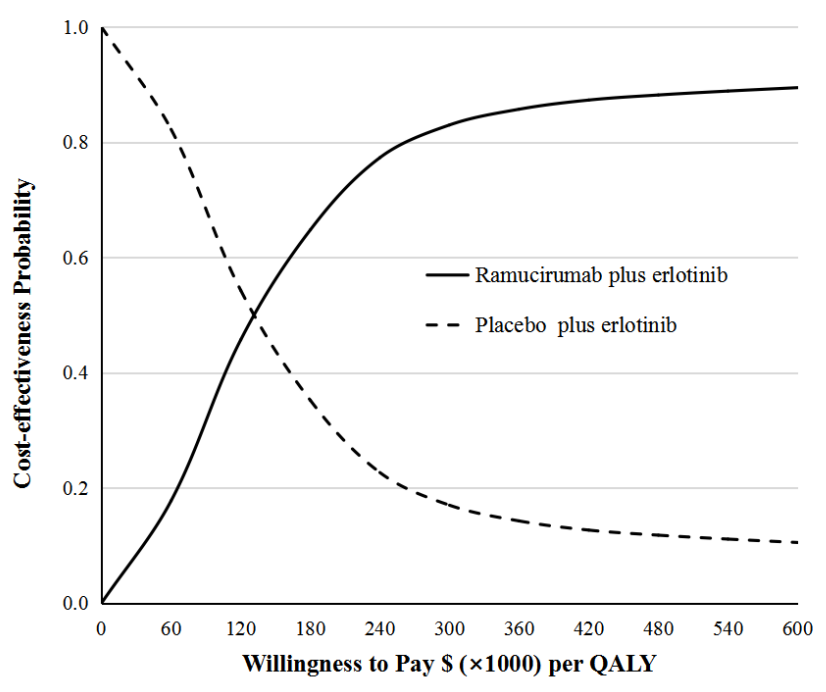

Figure 3 The cost-effectiveness acceptability curve. QALY, quality-adjusted life-year.

we set the WTP threshold for general regions ( $\$ 30363$, $3 \times$ national per-capita GDP in 2019) and affluent regions ( $\$ 70353,3 \times$ per-capita GDP of Beijing), respectively.

Although other dual inhibition of EGFR and VEGF pathways, such as bevacizumab plus erlotinib, has been recommended in EU and Japanese NSCLC treatment guidelines, ${ }^{16}$ we did not evaluate this therapy in comparison with ramucirumab plus erlotinib, because of the limitations of these published trials (small sample sizes, open-label designs and Japanese-only population). Most notably, bevacizumab plus erlotinib demonstrated superior PFS similar to ramucirumab plus erlotinib based on the results of the NEJ026. ${ }^{18}$ Considering that China has

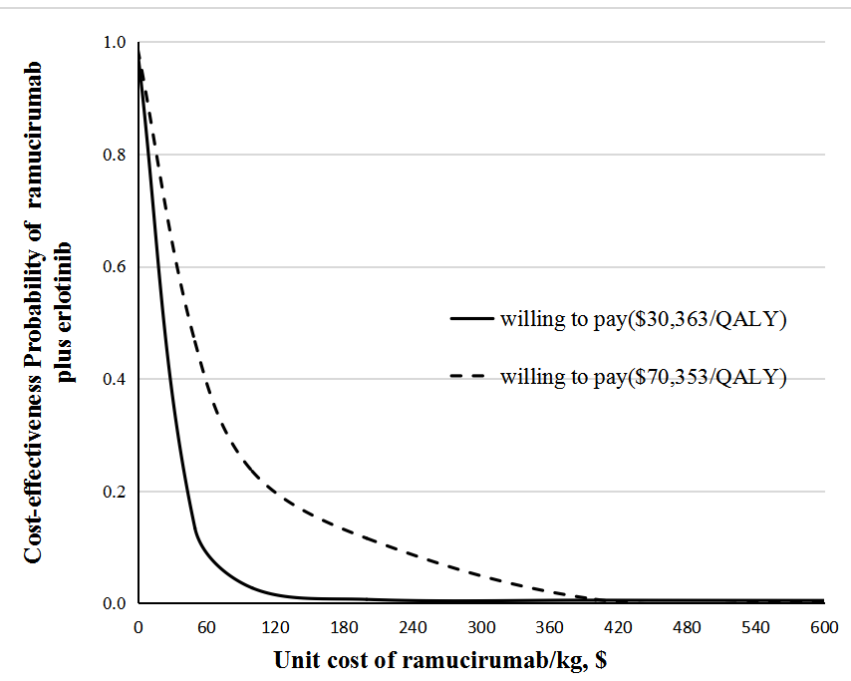

Figure 4 Probability of cost-effectiveness of ramucirumab plus erlotinib versus placebo plus erlotinib at different drug costs of ramucirumab. The dotted line represents the WTP threshold of $\$ 70$ 353/QALY for affluent regions, while the solid line represents the WTP threshold of \$30 363/QALY for general regions. QALY, quality-adjusted life-year; WTP, willingness-to-pay. the largest population of lung cancer in the world with limited resources, future phase III randomised trials are urgently needed to develop economic analyses of these innovative therapies.

However, this study has several limitations. First, just like all Markov models, our cost-effectiveness analysis was based solely on the phase III RELAY trial. Even more remarkable, this trial enrolled a predominantly Asian patient population (346 (77\%) of 449), but the current analysis should be interpreted with caution because this worldwide trial may not fully conform to the clinical pathway and treatment pattern in China. Second, costs data from various sources present potential uncertainty. In our model, the drug costs data were estimated based on the Chinese market price, while other costs obtained directly from published literature, which reported medical costs associated with Chinese patients with cancer, however, all efforts were made to handle the uncertainty of costs parameters by performing a series of sensitivity analyses. Third, the utilities data were captured from a previously published international study, any biases in this study will be reflected in the model. Although the sensitivity analyses suggested only small impacts of utilities on our results, Chinese-specific health utility value remains an open question. Fourth, at the time of data cut-off, the OS data of ramucirumab plus erlotinib and placebo plus erlotinib remain immature. According to the statistical goodness-of-fit, an exponential survival model was used to extrapolate the long-term OS beyond the follow-up duration of the RELAY trial. Although there were slight numerical differences in costs and QALYs between lifetime and 64 Markov cycles, which was the survival follow-up time in the RELAY trial, the differences with regard to economic outcomes were not significant. Nevertheless, when mature OS data are available, the current findings could be verified.

Overall, from the perspective of Chinese healthcare system, ramucirumab plus erlotinib strategy for patients with previously untreated EFGR-mutated mNSCLC without known EGFR Thr790Met mutation and CNS metastases was unable to be cost-effective compare to placebo plus erlotinib strategy. When the NRDL negotiation was available for ramucirumab, the ramucirumab plus erlotinib strategy might be a cost-effective therapeutic strategy in affluent regions in China.

Acknowledgements I certify that no individuals other than the listed co-authors contributed to this publication.

Contributors QL contributed to the study concepts and design; XZ, CT and XL contributed to the clinical program; LP, YL, XW and XL contributed to the analysis and interpretation of the data; $\mathrm{QL}, \mathrm{XZ}$ and $\mathrm{CT}$ contributed to the drafting and revising of the paper; all authors approved of final manuscript; all authors agree to be accountable for all aspects of the work.

Funding This work was supported by the Natural Science Foundation of Hunan Province (grant numbers 2019JJ50864); the project of scientific research plan of health and Health Commission of Hunan Province in 2019 (grant numbers B2019156).

Competing interests None declared.

Patient consent for publication Not required. 
Ethics approval Ethical approval was not necessary, because our economic evaluation is based on a mathematical model analysis, and does not contain any studies with human participants or animals performed.

Provenance and peer review Not commissioned; externally peer reviewed.

Data availability statement Data are available upon reasonable request.

Supplemental material This content has been supplied by the author(s). It has not been vetted by BMJ Publishing Group Limited (BMJ) and may not have been peer-reviewed. Any opinions or recommendations discussed are solely those of the author(s) and are not endorsed by BMJ. BMJ disclaims all liability and responsibility arising from any reliance placed on the content. Where the content includes any translated material, BMJ does not warrant the accuracy and reliability of the translations (including but not limited to local regulations, clinical guidelines, terminology, drug names and drug dosages), and is not responsible for any error and/or omissions arising from translation and adaptation or otherwise.

Open access This is an open access article distributed in accordance with the Creative Commons Attribution Non Commercial (CC BY-NC 4.0) license, which permits others to distribute, remix, adapt, build upon this work non-commercially, and license their derivative works on different terms, provided the original work is properly cited, appropriate credit is given, any changes made indicated, and the use is non-commercial. See: http://creativecommons.org/licenses/by-nc/4.0/.

ORCID iD

Chongqing Tan http://orcid.org/0000-0002-6007-4170

\section{REFERENCES}

1 Chen W, Zheng R, Baade PD, et al. Cancer statistics in China, 2015. CA Cancer J Clin 2016;66:115-32.

2 Chen WQ, Li H, Sun KX, et al. [Report of Cancer Incidence and Mortality in China, 2014]. Zhonghua Zhong Liu Za Zhi 2018;40:5-13.

3 Kilgoz HO, Bender G, Scandura JM, et al. Kras and the reality of personalized medicine in non-small cell lung cancer. Mol Med 2016;22:380-7.

4 Novello S, Barlesi F, Califano R, et al. Metastatic non-small-cell lung cancer: ESMO clinical practice guidelines for diagnosis, treatment and follow-up. Ann Oncol 2016;27:v1-27.

5 William WN, Lin HY, Lee JJ, et al. Revisiting stage IIIB and IV nonsmall cell lung cancer: analysis of the surveillance, epidemiology, and end results data. Chest 2009;136:701-9.

6 Hsu W-H, Yang JC-H, Mok TS, et al. Overview of current systemic management of EGFR-mutant NSCLC. Ann Oncol 2018;29:i3-9.

7 Park K, Tan E-H, O'Byrne K, et al. Afatinib versus gefitinib as first-line treatment of patients with EGFR mutation-positive non-small-cell lung cancer (LUX-Lung 7): a phase 2B, open-label, randomised controlled trial. Lancet Oncol 2016;17:577-89.

8 Soria J-C, Ohe Y, Vansteenkiste J, et al. Osimertinib in untreated EGFR-mutated advanced non-small-cell lung cancer. N Engl J Med 2018;378:113-25.

9 Wu Y-L, Cheng Y, Zhou X, et al. Dacomitinib versus gefitinib as firstline treatment for patients with EGFR-mutation-positive non-smallcell lung cancer (Archer 1050): a randomised, open-label, phase 3 trial. Lancet Oncol 2017;18:1454-66.

10 Yu HA, Arcila ME, Rekhtman N, et al. Analysis of tumor specimens at the time of acquired resistance to EGFR-TKI therapy in 155 patients with EGFR-mutant lung cancers. Clin Cancer Res 2013;19:2240-7.

11 Soria J-C, Ramalingam SS. Osimertinib in EGFR mutation-positive advanced NSCLC. N Engl J Med 2018;378:1262-3.

12 Takeda M, Nakagawa K. First- and second-generation EGFR-TKIs are all replaced to osimertinib in chemo-naive EGFR mutationpositive non-small cell lung cancer? Int J Mol Sci 2019;20:146.

13 Doroshow DB, Sanmamed MF, Hastings K, et al. Immunotherapy in non-small cell lung cancer: facts and hopes. Clin Cancer Res 2019;25:4592-602.

14 Cavanna L, Citterio C, Orlandi E. Immune checkpoint inhibitors in EGFR-mutation positive TKI-treated patients with advanced non-small-cell lung cancer network meta-analysis. Oncotarget 2019;10:209-15.
15 Reckamp KL. Targeted therapy for patients with metastatic non-small cell lung cancer. J Natl Compr Canc Netw 2018;16:601-4.

16 Planchard D, Popat S, Kerr K, et al. Metastatic non-small cell lung cancer: ESMO clinical practice guidelines for diagnosis, treatment and follow-up. Annals of Oncology 2018;29:iv192-237.

17 Byers LA, Heymach JV. Dual targeting of the vascular endothelial growth factor and epidermal growth factor receptor pathways: rationale and clinical applications for non-small-cell lung cancer. Clin Lung Cancer 2007;8 Suppl 2:S79-85.

18 Saito H, Fukuhara T, Furuya N, et al. Erlotinib plus bevacizumab versus erlotinib alone in patients with EGFR-positive advanced nonsquamous non-small-cell lung cancer (NEJ026): interim analysis of an open-label, randomised, multicentre, phase 3 trial. Lancet Oncol 2019;20:625-35.

19 Herbst RS, Ansari R, Bustin F, et al. Efficacy of bevacizumab plus erlotinib versus erlotinib alone in advanced non-small-cell lung cancer after failure of standard first-line chemotherapy (beta): a double-blind, placebo-controlled, phase 3 trial. Lancet 2011;377:1846-54.

20 Johnson BE, Kabbinavar F, Fehrenbacher L, et al. Atlas: randomized, double-blind, placebo-controlled, phase IIIB trial comparing bevacizumab therapy with or without erlotinib, after completion of chemotherapy, with bevacizumab for first-line treatment of advanced non-small-cell lung cancer. J Clin Oncol 2013;31:3926-34.

21 Spratlin JL, Cohen RB, Eadens M, et al. Phase I pharmacologic and biologic study of ramucirumab (IMC-1121B), a fully human immunoglobulin $\mathrm{G} 1$ monoclonal antibody targeting the vascular endothelial growth factor receptor-2. J Clin Oncol 2010;28:780-7.

22 Nakagawa K, Garon EB, Seto T, et al. Ramucirumab plus erlotinib in patients with untreated, EGFR-mutated, advanced non-small-cell lung cancer (relay): a randomised, double-blind, placebo-controlled, phase 3 trial. Lancet Oncol 2019;20:1655-69.

23 National Healthcare Security Administration. Notice on reimbursement decisions on including 17 cancer drugs in national reimbursement drug list by National healthcare security administration, 2018. Available: http://www.gov.cn/xinwen/2018-10/ 10/content_5328891.htm

24 Chinese Society of Clinical Oncology. Primary lung cancer treatment guidelines (2018 version), 2018. Available: http://www.csco.org.cn/ [Accessed 2020.11.21].

25 National Bureau of statistics of China. National Bureau of statisticsof China: China statistical yearbook 2019. Beijing: China Statistics Press, 2020.

26 Zeng X, Li J, Peng L, et al. Economic outcomes of maintenance gefitinib for locally advanced/metastatic non-small-cell lung cancer with unknown EGFR mutations: a semi-Markov model analysis. PLoS One 2014;9:e88881.

27 Nafees B, Lloyd AJ, Dewilde S, et al. Health state utilities in nonsmall cell lung cancer: an international study. Asia Pac J Clin Oncol 2017;13:e195-203.

28 Lu S, Yu Y, Fu S, et al. Cost-effectiveness of ALK testing and first-line crizotinib therapy for non-small-cell lung cancer in China. PLoS One 2018; 13:e205827.

29 Wu B, Dong B, Xu Y, et al. Economic evaluation of first-line treatments for metastatic renal cell carcinoma: a cost-effectiveness analysis in a health resource-limited setting. PLOS One 2012;7:e32530.

30 Zhang C, Zhang H, Shi J, et al. Trial-Based cost-utility analysis of Icotinib versus gefitinib as second-line therapy for advanced nonsmall cell lung cancer in China. PLoS One 2016;11:e151846.

$31 \mathrm{Wu} \mathrm{B}$, Li T, Cai J, et al. Cost-Effectiveness analysis of adjuvant chemotherapies in patients presenting with gastric cancer after D2 gastrectomy. BMC Cancer 2014;14:984.

32 Zeng X, Karnon J, Wang S, et al. The cost of treating advanced nonsmall cell lung cancer: estimates from the Chinese experience. PLOS One 2012; 7:e48323.

33 Guan H, Sheng Y, Guo W, et al. Cost-Effectiveness of alectinib for patients with untreated ALK-positive non-small cell lung cancer in China. Adv Ther 2019;36:1114-25.

34 Liu Q, Luo X, Peng L, et al. Nivolumab versus docetaxel for previously treated advanced non-small cell lung cancer in China: a cost-effectiveness analysis. Clin Drug Investig 2020;40:129-37. 